\title{
Comparison of $M$ and $T$ Type Antigen Testing to Field Inversion Gel Electrophoresis in the Differentiation of Strains of Group A Streptococcus
}

\author{
ROSY SHAH, MICHAEL GREEN, KAREN A. BARBADORA, WILLIAM C. WAGENER, BENJAMIN \\ SCHWARTZ, RICHARD R. FACKLAM, AND ELLEN R. WALD \\ Departments of Pediatrics [R.S., M.G., K.A.B., E.R.W.] and Surgery [M.G.], University of Pittsburgh \\ School of Medicine, and University of Pittsburgh Graduate School of Public Health [W.C.W.], Pittsburgh, \\ Pennsylvania, and Centers for Disease Control and Prevention [B.S., R.R.F.], Atlanta, Georgia
}

\section{ABSTRACT}

\begin{abstract}
Recent clusters of patients with acute rheumatic fever and invasive group A Streptococcus (GAS) have stimulated renewed interest in the epidemiology of streptococcal infections. We compared conventional serotyping for $\mathrm{M}$ and $\mathrm{T}$ antigens and serum opacity factor with field inversion gel electrophoresis (FIGE) for distinguishing among GAS. Fifteen pairs of throat isolates obtained from children positive for GAS before and after therapy were evaluated by conventional serotyping and by FIGE after Smal digestion. Ten of the 15 pairs were identical by serotyping. FIGE correctly identified the 10 concordant and 5 discordant pairs. Individual clones were identified within each $M$ type tested, including analysis performed on additional isolates
\end{abstract}

GAS is responsible for a wide array of clinical manifestations including suppurative infections and nonsuppurative sequelae such as rheumatic fever and glomerulonephritis (1). The recent clusters of patients with acute rheumatic fever and invasive GAS have stimulated renewed interest in the epidemiology of streptococcal infections. New typing methods for the differentiation of various strains of streptococci have been described. One such method, pulsed-field electrophoresis, utilizes DNA RFLP to distinguish among strains of the same bacterial species. The purpose of this study was to compare conventional $\mathrm{M}$ and $\mathrm{T}$ type antigen testing with FIGE, a type of pulsed-field electrophoresis, for distinguishing among strains of GAS.

\section{METHODS}

Study isolates. Children with pharyngitis caused by GAS were enrolled at our center in an ongoing study comparing the

Received January 6, 1995; accepted June 14, 1995.

Correspondence: Michael Green, M.D., M.P.H., Division of Infectious Diseases, Children's Hospital of Pittsburgh, 3705 Fifth Ave., Pittsburgh, PA 15213.

Presented in Part at the 1994 Annual Meeting of the Society for Pediatric Research, Seattle, WA. of M1 and M3 obtained from the Centers for Disease Control and Prevention. This preliminary experience suggests that FIGE can successfully determine whether serial isolates from a given patient represent persistence of one strain or acquisition of a new strain of GAS and that this method might provide an alternative typing system for GAS. (Pediatr Res 38: 988-992, 1995)

\section{Abbreviations}

GAS, group A streptococcus

FIGE, Field inversion gel electrophoresis RFLP, restriction fragment length polymorphorism OF, opacity factor therapeutic efficacy of penicillin and cefadroxil. Cultures were performed before and after treatment. Organisms were confirmed to be GAS by the Streptex method (Wellcome, Dartford, UK). Fifteen patients who had positive cultures 3-5 d after completion of antibiotic therapy were labeled "bacteriologic failures," and their paired isolates were sent to the Centers for Disease Control and Prevention (CDC) in Atlanta, $\mathrm{GA}$, for $\mathrm{M}$ and $\mathrm{T}$ typing (2) as well as determination of serum OF.

In addition, nine isolates of type M1 and 10 isolates of type M3 GAS from diverse geographic areas within the United States were obtained from the CDC for evaluation by FIGE. Strains within these two sets of bacteria were epidemiologically unrelated.

Isolation of DNA in agarose plugs. After overnight growth in $10 \mathrm{~mL}$ of Todd-Hewitt broth (Becton Dickinson, Cockeysville, MD) at $37^{\circ} \mathrm{C}$, bacteria were centrifuged, and the resulting pellet was suspended in $5 \mathrm{~mL}$ of PIV buffer $(1 \mathrm{M}$ $\mathrm{NaCl}, 10 \mathrm{mM}$ Tris-HCl, $\mathrm{pH} 7.6$ ). An aliquot of $250 \mu \mathrm{L}$ of $3.2 \%$ low melting agarose (Sigma Chemical Co., St. Louis, MO) was added to $750 \mu \mathrm{L}$ of the bacterial suspension (resulting in a final agarose concentration of $0.8 \%$ ), and plugs were made in a 
DNA sample mold (Bio-Rad, Hercules, CA). After solidification, the plugs were incubated overnight at $37^{\circ} \mathrm{C}$ in $5 \mathrm{~mL}$ of freshly prepared lysis buffer $(1 \mathrm{M} \mathrm{NaCl}, 100 \mathrm{mM}$ EDTA, pH 7.5, 6 mM Tris- $\mathrm{HCl}$, pH 7.6, 0.5\% Brij 58, $0.2 \%$ deoxycholate, $0.5 \%$ Sarkosyl, $20 \mu \mathrm{g} / \mathrm{mL}$ RNase A, $2 \mathrm{mg} / \mathrm{mL}$ lysozyme). Lysis buffer was removed and replaced with freshly prepared ESP buffer (0.5 M EDTA, pH 9-9.5, 10\% Sarkosyl, $100 \mu \mathrm{g} / \mathrm{ml}$ proteinase $\mathrm{K}$ ) for overnight incubation at $50^{\circ} \mathrm{C}$. ESP was then removed and the plugs were washed three times with TE buffer(1 M Tris- $\mathrm{HCl}, \mathrm{pH} 8,0.25 \mathrm{M}$ EDTA) at $37^{\circ} \mathrm{C}$ for $30-\mathrm{min}$ intervals and stored at $4{ }^{\circ} \mathrm{C}$.

Restriction enzyme digestion. Three 4-mm slices of the plugs were digested with $24 \mathrm{U}$ of SmaI, ApaI and SfiI enzymes in their respective $1 \times$ restriction buffers (New England Biolabs, Beverly, MA) and placed at room temperature (SmaI and $A p a \mathrm{I})$ and at $50^{\circ} \mathrm{C}(\mathrm{SfiI})$ for at least $16 \mathrm{~h}$. Excess restriction buffer was suctioned off. The plugs were melted in a waterbath at $65^{\circ} \mathrm{C}$ and loaded into wells of a $1 \%$ agarose gel (SeaKem LE Agarose, FMC Bioproducts, Rockland ME) along with the $\lambda$ ladder from Saccharomyces cerevisiae (New England Biolabs) for DNA size standards.

FIGE. FIGE was performed at $4-8^{\circ} \mathrm{C}$ using a Switchback Pulse Controller (Hoefer Scientific Instruments, San Francisco, CA) with a voltage gradient of $8 \mathrm{~V} / \mathrm{cm}$. Conditions for FIGE included an initial forward pulse of $0.6 \mathrm{~s}$, a final forward pulse of $17.4 \mathrm{~s}$, a ratio of forward to reverse pulse duration of $3: 1$, and a total run time of $40 \mathrm{~h}$. The gels were then stained with ethidium bromide and photographed under an UV light source.

Interpretation of FIGE. Migration of DNA fragments was compared with that of the $\lambda$ ladder DNA standards. Bands falling in the $50-250-\mathrm{kb}$ window were used for the analysis. Interpretation of clonal relationships was performed according to the recent proposal of Goering (3). Isolates were considered identical if there was complete agreement between the band patterns. Isolates were categorized as clonally related if there was no more than a three-band difference between patterns (3). Finally, isolates were considered clonally unrelated if there was more than a three-band difference between them.

\section{RESULTS}

Initial evaluation was performed using SmaI, ApaI and SfiI on eight separate clinical isolates (not shown). SmaI was found to produce an average of 10 bands in the $50-250-\mathrm{kb}$ range. ApaI produced a similar but less distinct number of bands, whereas Sfil produced only about four or five bands in this window. SmaI was chosen for the remainder of the analysis in this study.

Ten pairs of GAS isolates collected before and after antibiotic treatment were found to be identical by $\mathrm{M}$ and $\mathrm{T}$ typing (Table 1) indicating that the original pharyngeal strain persisted despite therapy or that the patients were carriers of GAS. The remaining five patients had different $\mathrm{M}$ and $\mathrm{T}$ type strains recovered before and after treatment, indicating loss of the original strain and acquisition of a new strain of GAS.

FIGE correctly identified the 10 concordant and five discordant pairs (Fig. 1). In the latter case, differences between the
Table 1. Results of $M$ and T typing and determination of $O F$ isolates of GAS recovered before and after antibiotic treatment

\begin{tabular}{llccc}
\hline Patient & Isolate & M type & T type & OF \\
\hline MH & Before & NT & 3 & - \\
& After & NT & 3 & - \\
UH & Before & 3 & 3 & - \\
& After & 3 & 3 & - \\
SJ & Before & NT & 3 & - \\
& After & NT & 3 & - \\
CL & Before & 12 & 12 & - \\
& After & 12 & 12 & - \\
AM & Before & NT & 4 & + \\
& After & NT & 4 & + \\
FM & Before & 3 & 3 & - \\
& After & 3 & 3 & - \\
MP & Before & 12 & 12 & - \\
& After & 12 & 12 & - \\
SS & Before & NT & $5 / 27 / 44$ & - \\
& After & NT & $5 / 27 / 44$ & - \\
AU & Before & NT & $5 / 27 / 44$ & - \\
& After & NT & $5 / 27 / 44$ & - \\
MV & Before & 3 & 3 & - \\
& After & NT & $11 / 12$ & + \\
KW & Before & NT & 6 & - \\
KF & After & NT & 6 & - \\
& Before & NT* & 4 & + \\
JH & After & 3 & 3 & - \\
& Before & NT & 12 & - \\
CR & After & NT & 28 & + \\
& Before & NT & 28 & + \\
JV & After & $12 \dagger$ & 12 & - \\
& Before & 3 & 3 & - \\
& After & NT & $11 / 12$ & + \\
\hline
\end{tabular}

* Nontypeable.

$\dagger$ Initially identified as M nontypeable. Found to be M12 on repeat testing.

pairs always exceeded three bands indicating that the pairs were not clonally related.

Six GAS isolates of type M3, T3, OF negative from four patients were compared. Four of these had identical patterns, and the other two (obtained from the same patient) differed from the rest by only one band (Fig. 2, lanes 7 and 8), indicating that these isolates are clonally related. Similarly, a comparison of four isolates (two each from two patients) serotyped as $\mathrm{M}$ nontypeable, $\mathrm{T} 5 / 27 / 44$, OF negative, revealed identical patterns within the pairs (Fig. 2, lanes 9 with 10 and lanes 11 with 12). However, a difference of five bands between the pairs was noted. This probably means that these strains were two different $M$ types with the same $T$ type pattern (i.e. M5 and M27 are both T 5/27/44, OF negative).

FIGE was performed under identical conditions on 10 type M3 isolates of GAS obtained from the CDC. Nine of these isolates had identical band patterns (Fig. 3). The tenth isolate differed from the rest by one band, indicating that these strains are all clonally related. Similarly, Figure 4 shows a comparison of nine M1 isolates obtained from different geographic locations in the United States. Although there was more heterogenitity among the electrophoretic patterns from these M1 strains than that seen among M3 strains, they differed from each other by no more than three bands. In contrast, they differed by more than three bands from each of the other serotypes evaluated in this study. Thus, these isolates also appear to be clonally related. 


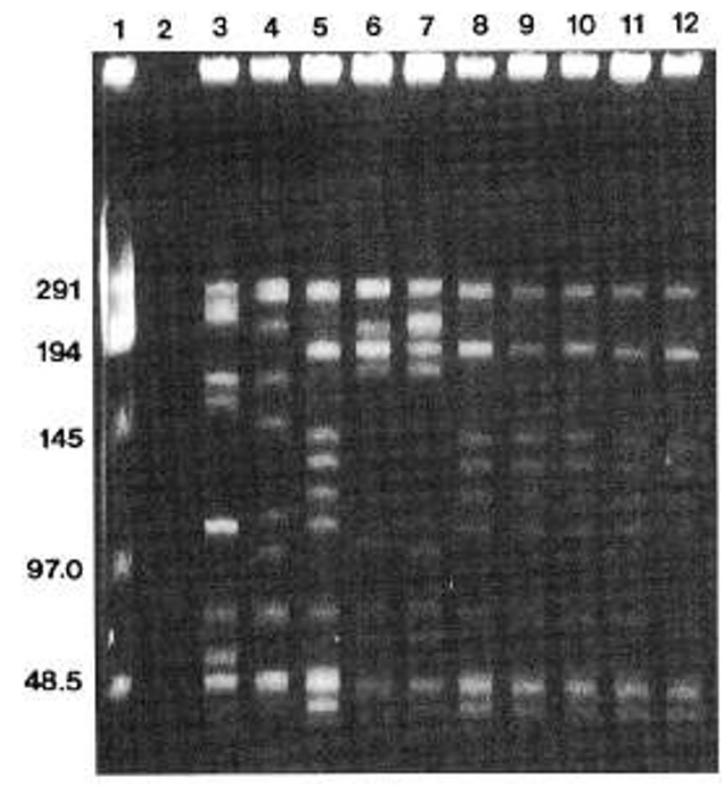

Figure 1. Typical FIGE analysis for GAS after digestion with SmaI. Lane 1, $\lambda$ DNA concatemers (from S. cerevisiae) expressed in kb. Lane 3, M NT, T4, OF-; lane 4, M3, T3, OF-; lane 5, M NT, T12, OF-; lane 6, M NT, T28, OF-; lane 7, M NT, T28, OF+; lane 8, M NT, T11/12, OF-; lane 9, M12, T12, OF-; lane 10, M12, T12, OF-; lane 11, M12, T12, OF-; lane 12, M12, $\mathrm{T} 12, \mathrm{OF}-$. Discordant pairs from three patients are shown in lanes 3 and 4, 5 and 6 , and 7 and 8 . Concordant pairs from two patients are seen in lanes 9 through 12 .

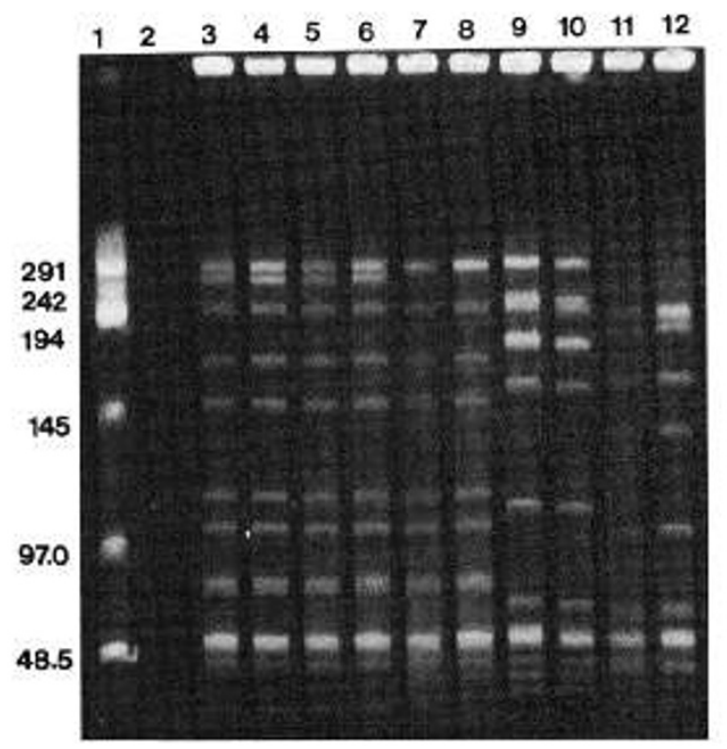

Figure 2. Results of FIGE on 10 isolates from this study. Lane 1, $\lambda$ DNA concatemers (from $S$. cerevisiae) expressed in $\mathrm{kb}$. Isolates in lanes 3 through 8 were typed $\mathrm{M} 3, \mathrm{~T} 3, \mathrm{OF}-$. A difference of only one band was seen between these isolates, indicating that these strains are all clonally related. Lanes 9 through 12 were four isolates of M nontypeable, T 5/27/44, from two patients. Identical patterns were seen in each of the two isolates recovered from the same patient (lanes 9 with 10 and lanes 11 with 12) but a five-band difference was noted between the two pairs.

\section{DISCUSSION}

Although the frequency of streptococcal pharyngitis has remained essentially unchanged over the past five decades, the severity of infections and the frequency of nonsuppurative

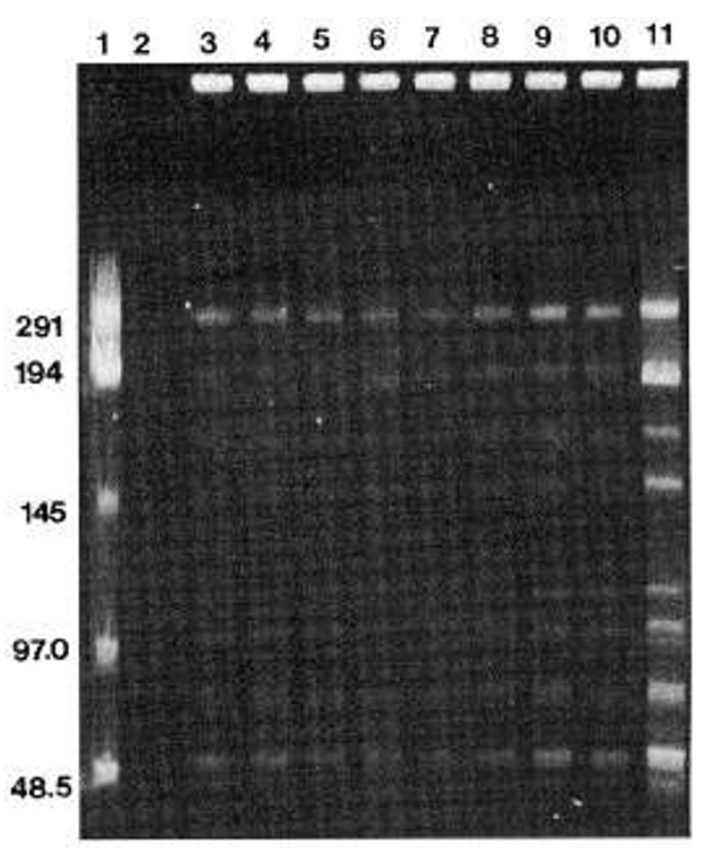

Figure 3. Results of FIGE on $10 \mathrm{M} 3$ isolates of GAS obtained from the CDC. Lanes 1 and 11, $\lambda$ DNA concatemers (from $S$. cerevisiae) expressed in $\mathrm{kb}$. Lanes 2-10, isolates identified as M3 by CDC.

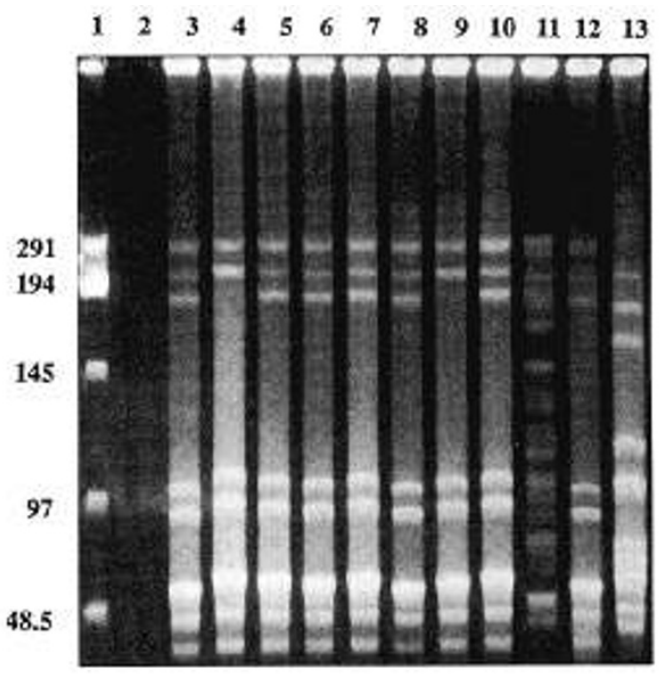

Figure 4. Results of FIGE for M1 isolates obtained from the CDC. Lane 1, $\lambda$ DNA concatemers (from S. cerevisiae) expressed in kb. Lanes 3 through 10 and lane $12, \mathrm{M} 1$ strains from the CDC. The pattern occurring most frequently is seen in lanes 3, 5 through 8, 10, and 12 and was labeled I. FIGE patterns of isolates in lanes 4 and 9 differ from I by one band and was labeled $\mathrm{I}^{\prime}$. Lane 11, isolate initially sent as "M1" but identified by FIGE as M3. Repeat serotyping by the CDC verified isolate as M3. Lane 13, isolate originally sent as "M3" with FIGE pattern unrelated to any of our other isolates. Repeat serotyping identified this strain as M13, T3/13, OF+

complications (especially rheumatic fever) has been variable. In the 1980s, a new wave of severe and invasive GAS infections occurred characterized by the recognition and increasing occurrence of a toxic shock-like syndrome accompanied in some cases by myositis, fasciitis, and deep tissue infections (4-6). Simultaneously, a resurgence of patients with rheumatic fever were documented in several parts of the United States and Europe (7). This increase in the frequency of severe GAS 
infections has been associated with an increased proportion of of certain serotypes of GAS that often produce mucoid colonies on blood agar plates; many of these isolates produce streptococcal pyrogenic exotoxins, in particular toxin $\mathrm{A}$, which had been uncommon for many years (8). $M$ types 1 and 3 in particular showed significant increases during this period and were more likely to be associated with invasive and sometimes fatal infections (9).

These clinical phenomena have evoked renewed interest in the epidemiology and pathogenesis of streptococcal infections. Serologic classification systems based primarily on the presence of specific $\mathrm{M}$ and $\mathrm{T}$ protein antigens (10) and detection of OF production (11) remain the "gold standard" for accurate identification of GAS in epidemiologic studies (2). Since the first recognition of the $M$ protein, approximately 80 antigenically different types have been described. However, there are several shortcomings to traditional serotyping. Many GAS isolates are currently not $\mathrm{M}$ protein typeable because of either poor sensitivity or a lack of availability of appropriate $M$ antisera. Currently, antisera are available only for $42 \mathrm{M}$ types at the CDC. More than $50 \%$ of isolates evaluated annually in their laboratory are considered $\mathrm{M}$ nontypeable. Accordingly, when a strain is found to be $M$ nontypeable, $T$ typing and/or OF analysis is used to trace the epidemiology of infection.

$\mathrm{M}$ antisera are laborious and expensive to prepare because of poor immunogenicity of some $M$ proteins. Thus, the small number of reference laboratories performing $\mathrm{M}$ typing use their own antisera, making comparison of serotyping results between laboratories difficult. Additionally, $M$ typing is dependent on phenotypic expression of the M protein, which may be affected by environmental growth conditions. Thus experimental conditions may influence the identification of a strain as $M$ nontypeable. Finally, preliminary comparisons of alternate molecular methods with $\mathrm{M}$ typing suggest that serotyping may be insufficient for clone differentiation (12).

Limited experience with several alternative typing systems has been reported, including phenotypic methods such as phage typing (13), bacteriocin typing (14), and multilocus enzyme electrophoresis (15). However, these methods have not found widespread use. Genomic typing methods include restriction endonuclease analysis and analysis of DNA RFLP of rRNA genes (ribotyping) (12, 16, 17). DNA RFLP using frequent cutting enzymes such as HindIII have been used as a tool for the evaluation of treatment failures in patients with pharyngitis caused by GAS (17). However, this enzymatic digestion produces a large number of bands making comparison of different strains cumbersome. Using this method, others have demonstrated that DNA fingerprints of GAS are $M$ type-specific and may be used to distinguish epidemiologically distinct strains within the same serotype (12). More recently the direct use of oligonucleotide probes for $\mathrm{N}$-terminal regions of the $\mathrm{M}$ protein gene ( $\mathrm{emm}$ genes) has been investigated, and preliminary results have been promising (18).

Pulsed-field gel electrophoresis of chromosomal DNA exposed to an infrequent cutting restriction endonuclease is another alternative for classifying streptococci. The use of SmaI restriction enzyme has been shown to be useful for Grampositive bacterial chromosomal DNA from Enterococcus fae- calis (19), Streptococcus pneumoniae (20), and group B streptococcus (21). Our initial analysis showed that it produced a manageable number of bands in the $50-250 \mathrm{~kb}$ window thereby permitting the assessment of epidemiologic relatedness of GAS. FIGE of Smal digested DNA of GAS in this study identified concordant and discordant pairs correctly as compared with conventional $\mathrm{M}$ and $\mathrm{T}$ typing. Thus we were able to differentiate between patients who failed treatment and those who acquired a new strain after therapy.

FIGE was also able to differentiate between pairs of isolates from different patients serotyped as M nontypeable, T 5/27/44, OF negative. These data suggest that FIGE may be superior to $\mathrm{T}$ typing for strains that are $\mathrm{M}$ nontypeable or have a $\mathrm{T}$ type pattern associated with more than one $M$ type antigen. Interestingly, the FIGE pattern of our isolates that were M nontypeable, T11/12, OF negative, and $\mathrm{M}$ nontypeable, T12, OF negative, were identical to the pattern produced by the isolate typed as M12, T12, OF negative (Fig. 1, lanes 5 and 8 with lanes 9 through 12). On repeat testing, the $M$ nontypeable $\mathrm{T} 11 / 12$ strain was identified as M12, whereas the M nontypeable, T12 strain remained nontypeable. This illustrates that repeat serotyping may be necessary to confirm that a given strain is in fact $M$ nontypeable. It also suggests that strains identified as a specific $M$ type by an alternate typing system might be characterized as $M$ nontypeable despite repeated performance of serotyping. Accordingly, FIGE might be more sensitive for determining the $M$ type of this isolate than conventional serotyping. The latter could be confirmed through the use of a specific emm gene probes (18).

One important advantage of serotyping is the established relationships between specific $M$ types and clinical syndromes. Evidence from this study suggests that FIGE might serve as a surrogate for $\mathrm{M}$ typing for this role as well. Analysis of FIGE patterns of multiple epidemiologically unrelated isolates of M3 and M1 GAS from geographically diverse areas identified single clones within a given $M$ type. Although the number of isolates evaluated to date is small, it may be that, as more isolates of other $\mathrm{M}$ types are subjected to SmaI enzyme digestion and subsequent FIGE, there will be a limited number of specific patterns identified within an $M$ type.

Our experience is in contrast to the results reported from New Zealand where FIGE was performed on 231 isolates of seven different $M$ types (22). These investigators reported polymorphisms not only between the $M$ types but also within some M types, including M1 and M3. They found, using SfiI as the restriction enzyme, six different RFLP patterns among 90 M1 isolates but $74 \%$ of these belonged to a single pattern (23). However, none of the six distinct RFLP patterns found among M1 strains matched electrophoretic patterns obtained from other serotypes of GAS, suggesting that sets of RFLP patterns obtained from a given $M$ type will be specific to that $M$ type.

There are several potential explanations for these observed differences. Very few bands were seen when using SfiI; therefore, the utility of this enzyme for analysis of GAS seems less than optimal. The authors did not provide their definition of clonal relationships. RFLP patterns obtained may have been considered unique if they differed from each other by one or more bands. In contrast, we used the proposed algorithm of 
Goering which identified strains with less than a three-band difference as being clonally related (3). Perhaps, reanalysis of their results using this rule might identify strains with different electrophoretic patterns as being clonally related. Alternatively, the differences may be due to our relatively small sample size and the possibility that we may have tested a sample of clonally related strains. Thus, if we had evaluated more strains we may have found results more similar to their experience.

In summary, limited experience with FIGE of GAS suggests that this method might provide an alternative typing system for GAS. It has correctly categorized paired isolates from 15 patients differentiating persistence of one strain from acquisition of a new strain of GAS. Preliminary data suggest that correlation of FIGE with serotyping results might allow for the subsequent prediction of the M type of GAS. To demonstrate equivalency of this or other alternative typing systems to traditional serotyping, a large database of FIGE patterns of multiple strains of many serotypes of GAS will need to be developed.

\section{REFERENCES}

1. Bisno AL 1991 Group A streptococcal infections and acute rheumatic fever. N Engl J Med 325:783-793

2. Moody MD, Padula J, Lizana D, Hall CT 1965 Epidemiologic characterization of group A streptococci by $\mathrm{T}$-agglutination and $\mathrm{M}$-precipitation tests in the public health laboratory. Health Lab Sci 2:149-622

3. Goering RV 1993 Molecular epidemiology of nosocomial infection: analysis of chromosomal restriction length fragment patterns by pulsed-field gel electrophoresis. Infect Control Hosp Epidemiol 14:595-600

4. Klein JO 1991 Re-emergence of virulent group A streptococcal infections. Pediat Infect Dis J 10:S3-S6

5. Cone LA, Woodard DR, Schlievert PM, Tomory GS 1987 Clinical and bacteriologic observations of a toxic shock-like syndrome due to Streptococcus pyogenes. N Engl J Med 317:146-149

6. Stevens DL, Tanner MH, Winship J, Swarts R, Ries KM, Schlievert PM, Kaplan E 1989 Severe group A streptococcal infections associated with a toxic shock-like syndrome and scarlet fever toxin A. N Engl J Med 321:1-7
7. Veasy LG, Weidmeier SE, Orsmond GS, Ruttenberg HD, Boucek MM, Roth SJ, Tait VF, Thompson JA, Daly JA, Kaplan EL, Hill HR 1987 Resurgence of acute rheumatic fever in the intermountain area of the United States. N Engl J Med 316:421-427

8. Kaplan EL, Johnson DR, Cleary PP 1989 Group A streptococcal serotypes isolated from patients and sibling contacts during the resurgence of rheumatic fever in the United States in the mid 1980s. J Infect Dis 159:101-103

9. Ferrieri P 1991 Microbiologic features of current virulent strains of group A streptococci. Pediatr Infect Dis J 10:S20-S24

10. Lancefield RC 1933 A serological differentiation of human and other groups of hemolytic streptococci. J Exp Med 57:571-595

11. Maxted WR, Widdowson JP, Fraser CAM, Ball LC, Bassett DCJ 1973 The use of serum opacity reaction in the typing of group A streptococci. J Med Microbiol 6:83-90

12. Cleary PP, Kaplan EL, Livdahl C, Skjold S 1988 DNA fingerprints of Streptococcus pyogenes are $\mathrm{M}$ type specific. $\mathrm{J}$ Infect Dis 158:1317-1323

13. Skjold SA, Lewis WW 1976 Method for phage typing group A type 49 streptococci. J Clin Microbiol 4:232-238

14. Tagg JR, Bannister LV 1979 "Fingerprinting" $\beta$-hemolytic streptococci by their production of bacteriocine-like inhibitors. J Med Microbiol 12:397-411

15. Musser JM, Gray BM, Schlievert PM, Pichichero ME 1992 Streptococcus pyogenes pharyngitis: characterization of strains by multilocus enzyme genotype, $\mathrm{M}$ and $\mathrm{T}$ protein serotype and pyrogenic exotoxin gene probing. J Clin Microbiol 3:600-603

16. Cleary PP, Kaplan EL, Handley JP, Wlazlo A, Kim MH, Hauser AR, Schlievert PM 1992 Clonal basis for resurgence of serious Streptococcus pyogenes disease in the 1980s. Lancet 339:518-521

17. Bingen E, Denamur E, Lambert-Zechovsky N, Braimi N, El Lakany M, Elion J 1992 DNA restriction fragment polymorphism differentiates recurrence from relapse in treatment failures of Streptococcus pyogenes pharyngitis. J Med Microbiol 37:162164

18. Kaufhold A, Podbielski A, Johnson DR, Kaplan EL, Lutticken R 1992 M protein gene typing of Streptococcus pyogenes by nonradioactively labeled oligonucleotide probes. J Clin Microbiol 30:2391-2397

19. Murray, BE, Singh KV, Markowitz SM, Lopardo HA, Patterson JE, Zervos MJ, Rubeghio E, Eliopolus GM, Rice LB, Goldstein FW, Jenkins SG, Caputo GM, Nasnas $\mathrm{R}$, Moore LS, Wong ES, Weinstock G 1991 Evidence for clonal spread of a single strain of $\beta$-lactamase producing Enterococcus faecalis to six hospitals in five states. $\mathrm{J}$ Infect Dis 163:780-785

20. Lefevre JC, Faucon G, Sicard AM, Gasc AM 1993 DNA fingerprinting of Streptococcus pneumoniae strains by pulsed-field gel electrophoresis. J Clin Microbiol 31:2724-2728

21. Fasola E, Livdahl C, Ferrieri P 1993 Molecular analysis of multiple isolates of the major serotypes of group B streptococci. J Clin Microbiol 31:2616-2620

22. Single LA, Martin DR 1992 Clonal differences within M-types of the group A Streptococcus revealed by pulsed field gel electrophoresis. FEMS Microbiol Lett 70:85-90

23. Martin DR, Single LA 1993 Molecular epidemiology of group A Streptococcus M type 1 infections. J Infect Dis 167:1112-1117 